\title{
Are next-generation sequencing results knocking on Heaven's door for transplantation planning in chronic myelomonocytic leukemia?
}

\author{
Guillermo F. Sanz, ${ }^{1,2,3,4}$ Mariam Ibañez ${ }^{1,2,4,5}$ and Elvira Mora ${ }^{1,2}$ \\ ${ }^{1}$ Department of Hematology, Hospital Universitario y Politéenico La Fe, Valencia; ${ }^{2}$ Instituto de Investigación Sanitaria La Fe, Valencia \\ ${ }^{3}$ Department of Medicine, University of Valencia, Valencia; ${ }^{4}$ Centro de Investigación Biomédica en Red de Cáncer, CIBERONC, \\ Instituto de Salud Carlos III, Madrid and 'Departamento de Ciencias Biomédicas, Facultad de Ciencias de la Salud, Universidad CEU \\ Cardenal Herrera, Valencia, Spain
}

E-mail: GUILLERMO SANZ - sanz_gui@gva.es

doi:10.3324/haematol.2019.240853

C hronic myelomonocytic leukemia (CMML) is a heterogeneous malignant myeloid disorder included in the 2016 revision to the World Health Organization (WHO) classification of myeloid neoplasms and acute leukemia in the category of myelodysplastic syndromes/myeloproliferative neoplasms. CMML patients show a diverse biological, clinical picture and heterogeneous prognosis, with short overall survival (OS) and increased risk of progression to acute myeloid leukemia. The diagnosis of CMML requires a monocytosis, defined as an absolute monocyte count above $1 \times 10^{9} / \mathrm{L}$ that should represent $>10 \%$ of the white blood count (WBC) differential, which persists for more than 3 months and for which other causes of reactive monocytosis have been excluded. The current World Health Organization classification includes three CMML groups, divided on the basis of blast counts, for better prognostication: CMML-0 $[<2 \%$ peripheral blood (PB) and $<5 \%$ bone marrow (BM) blasts]; CMML-1 (2-4\% PB and/or 59\% BM blasts); and CMML-2 (5-19\% PB and/or 10-19\% BM blasts). The classical French-American-British classification, still widely used, divides CMML into so-called "dysplastic" CMML (WBC $\left.\leq 13 \times 10^{\circ} / \mathrm{L}\right)$ and "myeloproliferative" CMML (WBC >13 x10\%/L).

Cytogenetic abnormalities and somatic mutations are found in, respectively, $25-30 \%$ and up to $95 \%$ of CMML patients and both have a strong influence on OS. The validated CMML-specific cytogenetic risk classification recognizes three risk categories: low-risk (normal karyotype or loss of the Y chromosome as a single anomaly; 78\%), high-risk (trisomy 8 or abnormalities of chromosome 7 , or complex karyotype; $12 \%$ ), and intermediate-risk (all other abnormalities; 9\%). ${ }^{1}$ The most frequently mutated genes in CMML affect epigenetic regulation and DNA methylation (ASXL1 and TET2), RNA splicing (SRSF2), and transcription (RUNX1) and signaling pathways $(R A S) .{ }^{2-4}$ Frameshift and nonsense ASXL1 mutations confer an adverse prognosis, ${ }^{2-4}$ aggravated when EZH2 and ASXL1 mutations co-occur. ${ }^{5}$ DNMT3A and TP53 mutations, although less common in CMML, have also been associated with poorer OS. ${ }^{6,7}$ It is noteworthy that the number of mutations also influences patients' outcomes, as recently demonstrated in a study in which a shorter OS was observed in CMML patients with three or more concomitant mutations.

Specific prognostic scoring systems for individual risk assessment are essential in order to provide risk-adapted treatment. The most commonly used are the CMML-specific prognostic scoring system (CPSS), ${ }^{8}$ the MD Anderson Cancer Center prognostic score (MDAPS) ${ }^{9}$ and the revised International Prognostic Scoring System (IPSS$\mathrm{R})^{10}$ (the last only being applicable to "dysplastic" CMML). More recent scoring systems that also include somatic mutations are those by the Groupe Français des Myélodysplasies (GFM), ${ }^{2}$ the Mayo Clinic ${ }^{3}$, and the molecular CPSS. ${ }^{4}$ All these molecular prognostic scoring systems consider ASXL1 mutations; the molecular CPSS also takes into account mutations in NRAS, SETBP1, and RUNX1.

Allogeneic hematopoietic cell transplant (HCT) is the only potentially curative therapy for patients with CMML but the number of transplant-eligible patients is low because of these individuals' advanced age, comorbidities, and frailty. A recent multicenter retrospective study with 1,656 CMML patients of whom 89 received an allogeneic HCT demonstrated the benefit of HCT for patients with higher-risk disease as determined by the $\mathrm{CPSS}^{11}$ and multiple retrospective studies have documented a 3-year OS rate of 30-40\%. ${ }^{12-15}$ For high-risk transplant-ineligible and/or lower-risk patients the most widely used therapies are hydroxyurea, hypomethylating agents, and best supportive care. Recent evidence suggests that hypomethylating agents might be superior to hydroxyurea. ${ }^{11}$

Many studies have evaluated the prognostic factors for transplantation outcomes in CMML patients, with contradictory results. ${ }^{16}$ As would be expected, patients transplanted in complete remission ${ }^{13}$ as well as those with $<5 \%$ blasts at transplantation ${ }^{14}$ had better outcomes in comparison to those with more advance disease at transplantation. The favorable effect of using hypomethylating agents before transplantation over intensive chemotherapy is debatable. ${ }^{14-16}$ In a large study by the Center for International Blood and Marrow Transplantation Research (CIBMTR), the CPSS score at the time of HCT strongly influenced OS after transplantation. ${ }^{15}$ Table 1 shows the predictive factors for increased relapse or reduced OS evidenced in major studies on allogenic HCT for CMML.

In this issue of Haematologica, Woo and colleagues, analyze long-term outcomes after allogeneic HCT in 129 patients with CMML from a single institution and evaluate clinical and molecular risk factors associated with outcomes. ${ }^{17}$ Of note, this study is the first to evaluate the impact of somatic mutations determined by next-generation sequencing (NGS) on allogeneic HCT outcomes in a large and homogeneous series of patients from a single institution. In a subcohort of 52 patients in whom a NGS panel of 75 genes was used, $85 \%$ of patients had at least one mutation, congruent with previous reports on 
Table 1. Predictive factors for overall survival after allogeneic hematopoietic cell transplantation in relevant series of patients with chronic myelomonocytic leukemia.

\begin{tabular}{|c|c|c|c|c|}
\hline & $\begin{array}{c}\text { EBMT } \\
\left.\text { \{Symeonidis:2015 } 5^{13}\right\}\end{array}$ & $\begin{array}{c}\text { MDACC } \\
\text { \{Kongtim:2016 }\end{array}$ & $\begin{array}{c}\text { CIBMTR } \\
\text { \{Liu:2017 }\end{array}$ & $\begin{array}{c}\text { FHCRC } \\
\text { \{Woo:201917\} }\end{array}$ \\
\hline Study period & 1988-2009 & 1991-2013 & 2001-2012 & $1986-2017$ \\
\hline N. of patients & 513 & 83 & 209 & 129 \\
\hline $\begin{array}{l}\text { Median age, years } \\
\text { (range) }\end{array}$ & $53(18-75)$ & $57(18-78)$ & $57(23-74)$ & $55(7-74)$ \\
\hline Disease at HCT (CMML/AML, \%) & $56 / 44$ & $57 / 43$ & $100 / 0$ & $71 / 29$ \\
\hline
\end{tabular}

\section{Factors predicting OS}

Complete remission at HCT

Higher-risk categories by CPSS/MDAPS

Performance Status (KPS $\geq 90)$

HCT-Cl $\geq 4$

High-risk CMML-specific cytogenetics

Graft source (peripheral blood)

Transplant from matched related donors

Prior HMA treatment

Molecular profile

Favorable

Favorable

NA

NA

No effect

NA

No effect

Unfavorable/NA

Unfavorable/Unfavorable

NA

Favorable

NA

No effect
No effect
No effect
No effect
NA

No effect
NA

$\mathrm{NA}$

NA

Unfavorable

Favorable

Favorable

NA
No effect

NA

NA

Increased relapse:

-Mutations in NRAS, ATRX, WT1

$-\geq 10$ gene mutations

$\geq 4$ mutations in epigenetic regulators

\begin{tabular}{|c|c|c|c|c|}
\hline Conditioning intensity & No effect & No effect & NA & No \\
\hline Development of chronic GvHD & No effect & Favorable & NA & $\mathrm{NA}$ \\
\hline Age & No effect & No effect & NA & No effect \\
\hline Year of transplant & No effect & No effect & NA & No effect \\
\hline
\end{tabular}

EBMT: European Society for Blood and Marrow Transplantation; MDACC: MD Anderson Cancer Center; CIBMTR: Center for International Blood and Marrow Transplantation Research; FHCRC: Fred Hutchinson Cancer Research Center; HCT:: hematopoietic cell transplant; CMML; chronic myelomonocytic leukemia; AML: acute myeloid leukemia; OS: overall survival; CPSS: CMML-specific prognostic scoring system; MDAPS: MD Anderson prognostic scores; KPS: Karnofsky Performance Score; HCT-CI: Hematopoietic Cell Transplant-Comorbidity Index; PB: peripheral blood; HMA: hypomethylating agents, GvHD: graft-versus-host disease; NA: information not available.;

CMML. The most commonly mutated genes were ASXL1 (52\%), TET2 (42\%), and SRSF2 (25\%). Other frequently encountered mutations were evident for WT1 $(27 \%)$, RUNX1 (17\%), DNMT3A (17\%), SMC1A (17\%), EZH2 $(12 \%)$, and ATRX (12\%), highly likely because most patients had intermediate-2 or high-risk disease according to the CPSS. ${ }^{17}$

In the study by Woo et al., mutations in NRAS were associated with an increased relapse risk whereas mutations in ATRX and WT1, conferred both a higher relapse risk and inferior OS. Moreover, this study showed that a high overall mutation burden ( $\geq 10$ mutations) as well as the presence of four or more mutated epigenetic regulatory genes were linked to a higher risk of relapse. Unsupervised clustering revealed two higher-risk groups with specific associations between mutations and clinical features. The presence of a higher mutation burden was closely related to a longer period between diagnosis and transplantation but not with complex chromosomal abnormalities or an excess of blasts. The currently published recommendation of an international expert panel is to use the CPSS for considering a patient as a candidate for allogeneic HCT and to transplant those CMML patients belonging to the intermediate-2/high CPSS risk groups. ${ }^{18}$ Whether the better outcomes observed with lower CPSS scores and the lower mutational burden observed in less advanced disease could argue in favor of transplanting patients with CMML earlier in the course of their disease (e.g., extending transplantation to patients with intermediate-1 CPSS risk score) is debatable and can only be properly answered by a carefully designed study.

As previously reported in other HCT series on CMML, in the study by Woo et al., relapse risk was also significantly associated with adverse cytogenetics, higher-risk CPSS and MDAPS scores, and measurable residual disease by cytogenetics at transplantation. A higher mortality was seen in patients with high-risk cytogenetics and a high HCT Comorbidity Index. Of interest, neither disease status at transplantation (complete remission vs. non-complete remission) nor pre-transplant therapy (intensive chemotherapy or hypomethylating agents) nor conditioning intensity had a clear and independent impact on transplant outcomes. Additionally, the year of transplant did not affect the risk of relapse or OS, clearly indicating that newer transplant strategies are needed to improve those outcomes. ${ }^{17}$

In summary, this study has identified both clinical and novel molecular risk factors for outcomes after allogeneic HCT that add relevant information which could be taken into account when planning transplantation for CMML patients. Table 2 illustrates the clinical and molecular risk factors for allogeneic HCT outcome in CMML patients, among whom different groups of higher-risk patients amenable to transplantation can be detected at different 
Table 2. Schematic representation of the relationships between clinical and hematologic characteristics, cytogenetics, somatic mutations, prognostic scoring systems and transplant outcomes in patients with chronic myelomonocytic leukemia.

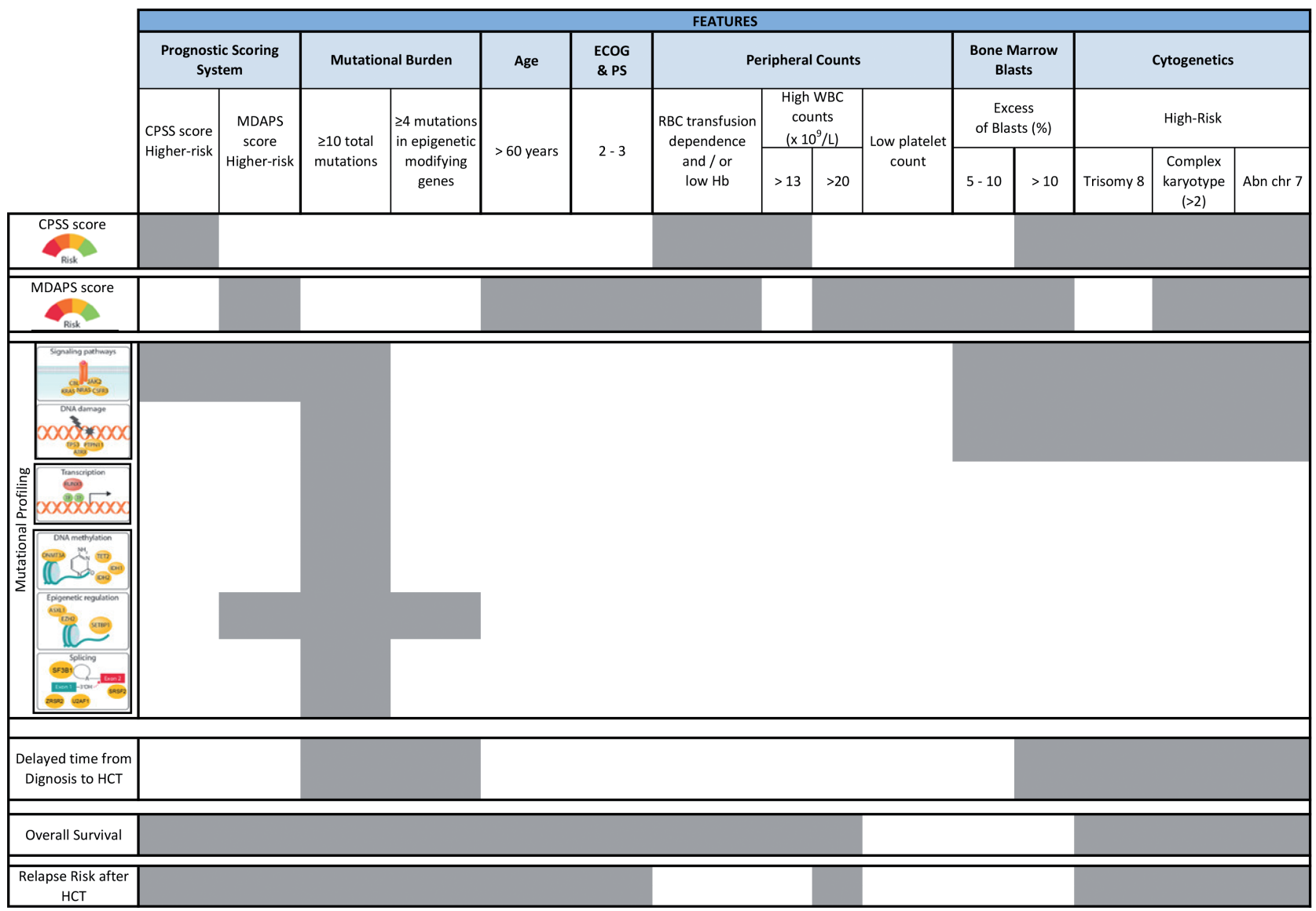

ECOG: Eastern Cooperative Oncology Group; PS: Performance Status; CPSS: CMML-specific prognostic scoring system; MDAPS: MD Anderson prognostic score; RBC: red blood cells; Hb: hemoglobin;WBC: white blood cells; Abn: abnormalities; Chr: chromosome; HCT: hematopoietic cell transplant

time points of the course of the disease.

However, even though NGS studies could therefore be used to select a group of high-risk CMML patients for transplantation, our view is that it might still be premature to incorporate the results of NGS techniques into the decision-making process for CMML patients undergoing allogeneic HCT. The main reasons are that NGS techniques and results are not well standardized, their reproducibility is unproven, the characterization of allele variants as pathogenic is not homogenously defined, and the variant allele frequency threshold used to define the presence of mutations is widely variable $5 \%$ in the series by Woo et al.) Furthermore, the impact of co-occurring mutations on prognosis is still unclear..$^{19}$ Additionally, the value of molecular profiling for treatment decision-making in CMML patients is diminished by the limited number of treatment alternatives and because there is no single somatic mutation that favors the use of a particular treatment approach. ${ }^{19}$

In conclusion, molecular profiling will likely emerge in the near future as highly valuable for planning transplantation in CMML patients, adding up to other well-recognized patient and disease characteristics such as higherrisk CPSS and MDAPS categories and HCT-Cormorbidity Index. Prospective cooperative studies focused on NGS results before and after transplantation and involving large numbers of patients will be eventually required to improve the cure rate afforded by allogeneic HCT in CMML.

\section{Acknowledgments}

This study was supported by research funding from Innovative Medicines Initiative 2 Joint Undertaking under HARMONY grant agreement n. 116026. This Joint Undertaking receives support from the European Union's Horizon 2020 Research and Innovation Program and EFPIA; FEDER funds (CIBERONC (CB16/12/00284)), "Fundación Española de Hematología (FEHH)"; "Instituto de Salud Carlos III" grants PI161011113, PI16/00665, and PI18/01472; and from the "Consellería de Educación, Cultura y Deporte" PROMETEOII/2015/008 and GVA/2018//004.

\section{References}

1. Such E, Cervera J, Costa D, et al. Cytogenetic risk stratification in chronic myelomonocytic leukemia. Haematologica. 2011;96(3):375383.

2. Itzykson R, Kosmider O, Renneville A, et al. Prognostic score including gene mutations in chronic myelomonocytic leukemia. J Clin Oncol. 2013;31(19):2428-2436.

3. Patnaik MM, Itzykson R, Lasho TL, et al. ASXL1 and SETBP1 muta- 
tions and their prognostic contribution in chronic myelomonocytic leukemia: a two-center study of 466 patients. Leukemia. 2014;28(11):2206-2212.

4. Elena C, Gallì A, Such E, et al. Integrating clinical features and genetic lesions in the risk assessment of patients with chronic myelomonocytic leukemia. Blood. 2016;128(10):1408-1417.

5. Patnaik MM, Vallapureddy R, Lasho TL, et al. EZH2 mutations in chronic myelomonocytic leukemia cluster with ASXL1 mutations and their co-occurrence is prognostically detrimental. Blood Cancer J. 2018;8(1):12

6. Patnaik MM, Barraco D, Lasho TL, et al. DNMT3A mutations are associated with inferior overall and leukemia-free survival in chronic myelomonocytic leukemia. Am J Hematol. 2017;92(1):56-61.

7. Montalban-Bravo G, Takahashi K, Patel K, et al. Impact of the number of mutations in survival and response outcomes to hypomethylating agents in patients with myelodysplastic syndromes or myelodysplastic/myeloproliferative neoplasms. Oncotarget. 2018;9(11):9714-9727.

8. Such E, Germing U, Malcovati L, et al. Development and validation of a prognostic scoring system for patients with chronic myelomonocytic leukemia. Blood. 2013;121(15):3005-3015.

9. Onida F, Kantarjian HM, Smith TL, et al. Prognostic factors and scoring systems in chronic myelomonocytic leukemia: a retrospective analysis of 213 patients. Blood. 2002;99(3):840-849.

10. Greenberg PL, Tuechler H, Schanz J, et al. Revised international prognostic scoring system for myelodysplastic syndromes. Blood. 2012;120(12):2454-2465.

11. Pleyer L, Leisch M, Kourakli A, et al. Effects of the therapeutic armamentarium on survival and time to next treatment in CMML subtypes: an international analysis of 950 cases coordinated by the AGMT study group. Blood. 2019;134(Suppl 1):844-844.
12. Park S, Labopin M, Yakoub-Agha I, et al. Allogeneic stem cell transplantation for chronic myelomonocytic leukemia: a report from the Societe Francaise de Greffe de Moelle et de Therapie Cellulaire. Eur J Haematol. 2013;90(5):355-364.

13. Symeonidis A, van Biezen A, de Wreede L, et al. Achievement of complete remission predicts outcome of allogeneic haematopoietic stem cell transplantation in patients with chronic myelomonocytic leukaemia. A study of the Chronic Malignancies Working Party of the European Group for Blood and Marrow Trans. Br J Haematol. 2015;171(2):239-246

14. Kongtim P, Popat U, Jiménez A, et al. Treatment with hypomethylating agents before allogeneic stem cell transplant improves progression-free survival for patients with chronic myelomonocytic leukemia. Biol Blood Marrow Transplant. 2016;22(1):47-53.

15. Liu HD, Ahn KW, Hu Z-H, et al. Allogeneic hematopoietic cell transplantation for adult chronic myelomonocytic leukemia. Biol Blood Marrow Transplant. 2017;23(5):767-775.

16. Sanz GF. A Lot to learn about allogeneic hematopoietic cell transplantation for chronic myelomonocytic leukemia. Biol Blood Marrow Transplant. 2017;23(5):713-714.

17. Woo J, Ro Choi D, Storer BE, et al. Impact of clinical, cytogenetic, and molecular profiles on long-term survival after transplantation in patients with chronic myelomonocytic leukemia. Haematologica. 2020;105(3):652-660

18. de Witte T, Bowen D, Robin M, et al. Allogeneic hematopoietic stem cell transplantation for MDS and CMML: recommendations from an international expert panel. Blood. 2017;129(13):1753-1762.

19. Sanz GF, Ibáñez M, Such E. Do next-generation sequencing results drive diagnostic and therapeutic decisions in MDS? Blood Adv. 2019;3(21):3454-3460.

\title{
Leukemia stem cell gene expression signatures contribute to acute myeloid leukemia risk stratification
}

\author{
Katherine L. B. Knorr and Aaron D. Goldberg
}

Division of Hematologic Malignancies, Department of Medicine, Memorial Sloan Kettering Cancer Center, New York, NY, USA

E-mail: AARON D. GOLDBERG - goldbera@mskcc.org

doi:10.3324/haematol.2019.241117

T he majority of patients with acute myeloid leukemia (AML) will die of their disease. Nevertheless, the prognosis of AML varies widely. Some AML patients may be cured by chemotherapy alone, while others require approaches such as allogeneic stem cell transplantation to have the best chance of longterm survival. As physicians, we are often asked by our AML patients: "How likely is this treatment going to work, and how long do I have to live?"1

Prognostication in AML has evolved over time. Initially, models for prediction of response to therapy were based on patient's parameters such as age and performance status in combination with cell characteristics such as morphology and chromosomal karyotype. With technological advancements, our understanding of disease biology has evolved and factors including molecular mutations and minimal residual disease have been integrated into prognostication schemes. Recently, an international expert panel on behalf of the European LeukemiaNet (ELN) published a revised version of a widely utilized prognostication scheme that categorizes AML patients into three risk groups (Favorable, Intermediate, and Adverse) based on genetic abnormalities (incorporating chromosomal karyotype and specific molecular muta- tions). ${ }^{2}$ These AML risk groups have profound clinical implications, particularly with regard to post-remission therapy for younger fit patients. In general, fit Favorablerisk AML patients who achieve a first complete remission after induction chemotherapy go on to consolidation chemotherapy with curative intent. However, even fit patients with Adverse- and Intermediate-risk AML are unlikely to be cured by chemotherapy alone, and therefore it is reasonable to consider allogeneic stem cell transplantation for Intermediate- and Adverse-risk patients upon achievement of first complete remission.

Why is AML so often resistant to chemotherapy? The biology of AML chemoresistance is complex. However, at a basic level, adverse-risk AML cells are more likely to evade conventional chemotherapeutics that target the cell cycle. It has therefore been hypothesized that one powerful driver of adverse prognosis in AML may be the properties of the leukemia stem cell (LSC), a type of cell that exhibits cell cycle quiescence, self-renewal, and chemoresistance. ${ }^{3-6}$ Although AML LSC remain challenging to isolate, assessment of AML LSC gene expression signatures has been proposed as a method to further refine prognosis - with LSC-like AML phenotypes contributing to adverse risk. 